\title{
Safety of Intravitreal Gene Therapy for Treatment of Subjects with Leber Hereditary Optic Neuropathy due to Mutations in the Mitochondrial ND4 Gene: The REVEAL Study
}

\author{
Catherine Vignal-Clermont ${ }^{1,2}$. Jean-François Girmens ${ }^{2,3} \cdot$ Isabelle Audo ${ }^{2,3,4} \cdot$ Saddek Mohand Said ${ }^{2,3,4}$. \\ Marie-Hélène Errera ${ }^{2,3,7} \cdot$ Lise Plaine $^{2,3} \cdot$ Denis $\mathrm{O}^{\prime}$ Shaughnessy ${ }^{5} \cdot$ Magali Taiel $^{5}$ (I) $\cdot$ José-Alain Sahel $^{3,4,6,7}$
}

Accepted: 16 January 2021 / Published online: 10 February 2021

(c) The Author(s) 2021

\begin{abstract}
Background Leber hereditary optic neuropathy (LHON) is a maternally inherited mitochondrial disease whose primary clinical manifestation is bilateral visual loss. Only a single therapy, idebenone, is approved in Europe for use in exceptional circumstances and no therapy is currently approved in the USA. LHON remains a disease with a high unmet medical need. Objective This is a report of an open-label, single-center, dose-escalation study that evaluated the safety and tolerability of lenadogene nolparvovec in 15 subjects with LHON for up to 5 years following a single intravitreal injection at four dose levels.

Methods Subjects were enrolled sequentially in four cohorts followed by an additional cohort at the dose selected, and safety was assessed by an independent data safety monitoring board (DSMB) prior to any dose escalation.

Results Overall, the treatment was well tolerated during the 5-year follow-up. No serious adverse events were considered related to treatment, no unexpected adverse events occurred, and no grade 3 or 4 Common Terminology Criteria for Adverse Events were reported. Anterior chamber inflammation and vitritis were mostly managed with topical steroids, and ocular inflammation was considered to be dose limiting by the DSMB based on the benefits/risks for the subjects. Analysis of the logarithm of the minimal angle of resolution (LogMAR) visual acuity in both treated and untreated eyes showed clinically relevant and durable improvements compared with baseline. Mean improvements of -0.44 and $-0.49 \operatorname{LogMAR}$ for treated and untreated eyes, respectively, were noted, with a mean ( \pm standard deviation) final value of LogMAR $+1.96 \pm 0.60$ and $+1.65 \pm 0.34$, respectively, at 5 years post-treatment administration. For the six subjects treated with the optimal dose level $\left(9 \times 10^{10}\right.$ viral genomes $[\mathrm{vg}] /$ eye $)$, the mean visual acuity improvement from baseline reached -0.68 LogMAR for treated eyes and $-0.64 \operatorname{LogMAR}$ for untreated eyes, with a mean final value of $\operatorname{LogMAR}+1.77 \pm 0.52$ and $+1.78 \pm 0.34$, respectively. While there was a meaningful improvement in visual acuity for REVEAL subjects, the final visual acuity was less favorable than that seen in the two subsequent pivotal phase III studies in which subjects were treated earlier during the course of their disease.
\end{abstract}

Conclusion Lenadogene nolparvovec was well tolerated with a good safety profile during 5 years of follow-up and may offer meaningful lasting improvements in vision for this LHON population.

Clinical Trial Number EUDRACT $\mathrm{N}^{\circ}$ 2013-001405-90.

Magali Taiel

mtaiel@GENSIGHT-BIOLOGICS.COM

1 Department of Neuro Ophthalmology and Emergencies, Rothschild Foundation Hospital, Paris, France

2 Centre Hospitalier National d'Ophtalmologie des Quinze Vingts, Paris, France

3 CHNO des Quinze Vingts, Institut Hospitalo Universitaire FOReSIGHT, INSERM-DGOS CIC 1423, Paris, France
4 Sorbonne Université, INSERM, CNRS, Institut de la Vision, 75012 Paris, France

5 GenSight Biologics, Paris, France

6 Fondation Ophtalmologique A. de Rothschild, 25-29 Rue Manin, 75019 Paris, France

7 Department of Ophthalmology, The University of Pittsburgh School of Medicine, Pittsburgh, PA, USA 


\section{Key Points}

Lenadogene nolparvovec (rAAV2/2-ND4; Lumevoq), a gene therapy for the treatment of Leber hereditary optic neuropathy due to mutation in the mitochondrial nicotinamide adenine dinucleotide dehydrogenase 4 , was studied in 15 subjects.

The treatment was well tolerated.

Efficacy signals were observed, with improved visual acuity in both treated and fellow eyes up to 5 years after a single treatment.

\section{Introduction}

Leber hereditary optic neuropathy (LHON) is a rare, maternally inherited mitochondrial genetic disease with a continued high unmet medical need. LHON is typically a nonsyndromic optic neuropathy primarily affecting the retinal ganglion cells (RGCs), whose axons form the optic nerve and extend into the brain via the optic chiasm and optic tract [1]. RGCs are located near the inner surface of the retina and receive visual information from photoreceptors via bipolar cell and retinal interneurons and collectively transmit image-forming and non-image-forming visual information. The pathophysiology of LHON is characterized by selective loss of RGCs and their axons, which leads to rapidly progressive bilateral visual loss.

LHON was the first inherited human disease associated with point mutations in the mitochondrial DNA (mtDNA) [2] and is considered the most common inherited genetic mitochondrial disorder [3]. Three primary point mutations in the mtDNA are responsible for LHON in approximately 90\% of subjects: G3460A, G11778A and T14484C, located, respectively, in the ND1, ND4 and ND6 genes. These genes code for nicotinamide adenine dinucleotide (NADH) dehydrogenases, subunits of complex I of the mitochondrial respiratory chain. These mutations in the mtDNA affect complex I subunits of the mitochondrial respiratory chain, causing impaired mitochondrial biogenesis and increased levels of reactive oxygen species. RGCs appear to be selectively vulnerable to mitochondrial dysfunction, resulting in apoptotic cell death, optic nerve degeneration and optic atrophy [4]. This manifests clinically with acute painless central visual loss, which is the hallmark of LHON [5].

The primary mutations are necessary but not sufficient to cause visual loss, and incomplete penetrance is well documented. It is estimated that approximately $50 \%$ of males and
$10 \%$ of females who carry one of the primary point mutations will manifest the clinical disease [6]. Genotype is the most significant prognostic factor of visual outcome, followed by age at onset of visual loss. Mutations in ND6 and ND4 are typically associated with the best and worst visual prognosis, respectively. Spontaneous recovery rates are highest among people with the T14484C ND6 mutation. On the other hand, the 11778-ND4 mutation is known to cause the most severe clinical form of LHON, with rare and poor visual spontaneous recovery in only approximately $4 \%$ of patients $[2,7,8]$. Moreover, it is well established that the natural history of the disease is better for young-onset patients (even with the 11778ND4 mutation), especially if they are aged $<15$ years [9-11].

LHON is a rare disease, with an estimated prevalence of between 1 in 30,000 and 1 in 50,000 reported in Northern Europe [4, 12, 13]. In the northeast of England, the reported prevalence of visual loss due to LHON is 3.22 per 100,000 people, and it is estimated that $2 \%$ of Australians aged $<65$ years who are registered as legally blind have visual loss from LHON [14]. The 11778-ND4 mutation is the most frequent, accounting for about $75 \%$ of LHON in North America and Europe [1, 4, 7].

Men are affected much more commonly than women, with a male predominance $>80 \%$ in most pedigrees [15]. Classically, subjects become affected between the age of 15 and 35 years, but onset of LHON has been reported at almost any age, from 2 to 87 years, in both men and women [14, 16, 17].

Newman et al. [18] conducted a meta-analysis of 204 subjects with ND4 LHON aged $\geq 15$ years at disease onset and revealed that only $11.3 \%$ of subjects experienced a certain level of spontaneous visual recovery. The authors could not rule out that treatment with idebenone had contributed to the improvement. This meta-analysis showed that the ND4 mutations are known to cause the most severe clinical form of LHON; importantly, among patients aged $\geq 15$ years, recovery of vision from nadir is poor and uncommon, and ultimate visual acuities of better than 20/200 are rare.

Therapeutic options for adolescent and adult patients with LHON are currently limited to idebenone (Raxone ${ }^{\circledR}$ ), a synthetic analog of coenzyme Q10, which is approved only in Europe under exceptional circumstances for treatment of LHON [19]. The RHODOS study [20] showed only a modest visual improvement of 7 Early Treatment Diabetic Retinopathy Study (ETDRS) letters in idebenone-treated subjects compared with placebo at 24 weeks. There is currently no approved treatment for LHON in the USA.

\section{Materials and Methods}

The REVEAL study, a phase I/IIa open-label, single-center, dose-escalation clinical trial, enrolled 15 subjects with LHON due to 11778 mutation in the mitochondrial NADH 
dehydrogenase 4 . Subjects were enrolled sequentially to four dose cohorts, each comprising three subjects $\left(9 \times 10^{9}\right.$ [cohort 1], $3 \times 10^{10}$ [cohort 2], $9 \times 10^{10}$ [cohort 3] and $1.8 \times 10^{11}$ [cohort 4] viral genomes [vg]/eye), and a fifth cohort (cohort 5) served as an expansion cohort at the dose selected for further development. The REVEAL study was designed to establish the safety and tolerability of lenadogene nolparvovec intravitreal injection (IVI) and to assess the best dose level to be implemented in pivotal phase III studies.

Subjects who met the eligibility criteria were enrolled sequentially. There was a 4-week waiting period after treatment of the first subject in each cohort and a further 4-week safety interval following treatment of the third subject in each cohort; the study data were then reviewed by the data safety monitoring board (DSMB) before escalation to the next dose level.

Subjects who had signed written informed consent were required to be aged $\geq 18$ years with a documented diagnosis of LHON based on a genetic test confirming the presence of the G11778A mutation in the mitochondrial ND4. The study eye had to have the worst vision of the two eyes, which was required to be stable for the previous 3 months and be $\leq 20 / 200$ for the first four cohorts and $\leq 20 / 63$ for the expansion cohort. No delay was imposed between the onset of visual loss and treatment administration. The study eye needed to have a retinal nerve fiber layer (RNFL) thickness of $\leq 80 \mu \mathrm{m}$, usually corresponding to a long time course of the disease. Furthermore, the study eye had to have sufficient viable RGCs, based on optical coherence tomography (OCT) images analyzed by the investigator. Females of childbearing potential had to have a negative serum pregnancy test and agree to practice effective birth control for 18 months following treatment. Male subjects had to agree to use condoms for 6 months following treatment.

The exclusion criteria were as follows. Subjects could not have absence of vision in the fellow eye, glaucoma, diabetic retinopathy, macula edema, vitreoretinal disease, pathology of the retina or the optic nerve, retinal vein occlusion, narrow angles, optic neuropathy from other causes or any other disease that could affect visual function. Nor could they have allergy or hypersensitivity to any of the trial products, anemia, severe coagulopathy or cardiovascular disease, HIV infection, significant systemic illness or any significant laboratory abnormalities. Subjects could not present with a known mutation of other genes implicated in pathological retinal conditions, nor could they have received oral corticoids within the previous 14 days or idebenone within 7 days. Eye surgery or participation in another clinical trial during the previous 3 months was not permitted. Also excluded were patients with any other condition that, in the opinion of the investigator, could have compromised the safety or compliance of the subject or would preclude the subject from successful completion of the study and subjects who were unable or unwilling to comply with the protocol requirements.

Following determination of eligibility for the study, lenadogene nolparvovec was administered as a single IVI of $180 \mu \mathrm{L}$ into the study eye at the following doses: $9 \times 10^{9}$ (cohort 1 ), $3 \times 10^{10}$ (cohort 2), $9 \times 10^{10}$ (cohorts 3 and 5) and $1.8 \times 10^{11}$ (cohort 4 ) vg/eye. An anterior chamber paracentesis was performed aseptically under local anesthesia immediately before the IVI to prevent increased intraocular pressure (IOP) due to the volume of the injection. Subjects were then followed for safety and efficacy assessment 3 days after treatment and again at 1, 2, 4, 8, 12, 24, 36 and 48 weeks post-treatment. Subjects were also followed in the longer term at 1.5, 2, 2.5, 3, 4 and 5 years post-treatment.

The primary endpoint of the study was the overall incidence of adverse events (AEs) up to 5 years post experimental treatment with this investigational medicinal product evaluated for each dose level and for the study as a whole. Similarly, the incidence of serious AEs (SAEs) was monitored. The secondary endpoints included safety assessments (humoral and cellular responses to AAV2) and efficacy measurements (best corrected visual acuity [BCVA], contrast sensitivity, color vision, perimetry exams, pattern electroretinogram [pERG], pattern reversal and flash visual evoked potentials [VEPs], OCT).

Study assessments included general clinical examination; hematology and biochemistry laboratory parameters; ETDRS BCVA; contrast sensitivity (Pelli Robson); color vision (15 hue color test); ophthalmic examination, including slit lamp biomicroscopy, IOP and fundoscopy; perimetry exams, including Humphrey perimetry SITA 24-2, semiautomated (Octopus 900) kinetic perimetry and microperimetry; pERG; pattern and flash VEPs recorded according to the International Society of Clinical Electrophysiology of Vision [21, 22]; and imaging exams, including color fundus photos (Canon Retinograph CR-2 AF, Tokyo, Japan) and Heidelberg Spectralis OCT. In addition, humoral and cellular responses against lenadogene nolparvovec were assessed. AEs and SAEs were recorded throughout the study, and the DSMB was informed of all SAEs on an ongoing basis. BCVA was tested with the ETDRS charts when subjects retained the visual ability to see letters on the chart and were expressed in logarithm of the minimal angle of resolution (LogMAR). Subjects unable to see any letters on the chart were tested for their ability to count fingers (CF) or detect hand motion (HM). The distance at which subjects correctly read the chart or identified $\mathrm{CF}$ or $\mathrm{HM}$ was documented. $\mathrm{CF}$ and $\mathrm{HM}$ were converted to $\log \mathrm{MAR}$ for analyses. For CF, the LogMAR value was calculated using this equivalence: $\log M A R=-\log (20 / 200 \times 20 /[$ distance in $\mathrm{cm} \times 0.0328])$. For $\mathrm{HM}$, the following formula was applied: $\operatorname{LogMAR}=$ $-\log (20 / 2000 \times 20 /[$ distance in $\mathrm{cm} \times 0.0328])$. For subjects with vision reduced to light perception or no light 
perception, the applied correspondence was LogMAR +4 and +4.5 , respectively. The 5-year follow-up visit was used to analyze the mean change from baseline.

The small cohort of subjects in this study meant that formal statistical analyses were not possible; therefore, data analyses were mostly descriptive in nature. The incidence and severity of AEs for each body system were presented for each dose level and summarized overall. Summary descriptive statistics were presented for all continuous variables related to laboratory data for each dose level and for the study overall. Descriptive statistics were performed for visual test results and immune monitoring.

The protocol was reviewed and approved by an independent ethics committee. The study was conducted in accordance with the principles and requirements of the International Conference on Harmonization Good Clinical Practice. The DSMB closely reviewed study data to ensure the continued safe conduct of the trial and protection of subjects.

\section{Results}

\subsection{Demographics}

A total of 19 subjects were screened; four were excluded (three were screen failures and one withdrew consent). In total, 15 subjects received treatment in five cohorts (four dose-escalation cohorts and one expansion cohort). There were no medical or surgical history violations in any of the 15 subjects contraindicating participation in the study or treatment administration. The time between the onset of visual loss and treatment administration ranged from 0.6 to 22.2 years, with the mean \pm standard deviation (SD) time period being $5.9 \pm 7.3$ years. For cohorts 3 and 5, who received $9 \times 10^{10} \mathrm{vg} /$ eye, the mean time was $4.6 \pm 8.7$ years.

The distribution of subjects at baseline $(n=15)$ was 13 males and two females. The mean age of subjects was $47.9 \pm 17.2$ years. All subjects were injected with a single dose of lenadogene nolparvovec in their corresponding cohorts.

Among the 15 included subjects, 12 completed the 5-year follow-up period. Three subjects from the first dose cohort did not complete the 5-year follow-up: two withdrew consent at 48 weeks and year 4, respectively, and one died at year 3 . The disposition of trial subjects is presented in Fig. 1.

\subsection{Safety}

\subsubsection{Adverse Events}

Overall, 192 treatment-emergent AEs (TEAEs) involving all 15 subjects were reported.
Six SAEs occurred in five subjects and were unlikely to be related or were unrelated to the study drug and unrelated to the IVI procedure. Among these subjects, one death was related to one SAE (septic shock secondary to endocarditis), which was assessed as unrelated to the study drug or the IVI procedure. The other SAEs were non-cardiac chest pain, Leber plus pseudo Leigh, pneumonia, radius fracture and fall.

No general safety issues were related to the study drug or the IVI procedure.

No changes of clinical significance were noted by the investigator for any of the vital signs, and most of the laboratory parameters were within normal ranges. All laboratory abnormalities were minor, and none were grade 3 or $4 \mathrm{Com}-$ mon Terminology Criteria for Adverse Events, considered clinically relevant, or related to the study drug or treatment procedures by the investigator.

No TEAEs occurred that led to study discontinuation. The majority of TEAEs were of mild intensity; eight were of moderate intensity and eight were severe.

The AEs that were considered severe $(n=8)$ were as follows: septic shock (SAE), non-cardiac chest pain (SAE), Leber plus pseudo Leigh (SAE), pneumonia (SAE), radius fracture (SAE), fall (SAE), anterior chamber inflammation, and vitritis.

The most frequently reported TEAEs were local ocular events, with 91 of all 192 TEAEs being ocular. The most common ocular TEAEs were intraocular inflammation and elevation of IOP. The intraocular inflammation included anterior chamber inflammation ( 25 events in ten subjects) and vitritis (19 events in 11 subjects).

Anterior chamber inflammation and vitritis were mostly assessed as mild in severity, with anterior chamber cell grade of $1+$ or lower (according to Standardization of Uveitis Nomenclature Working Group criteria; scores range from 0 to $4+$, with lower scores indicating less cells visible in the anterior chamber and lower severity of uveitis) [23] or vitreous haze grade $1+$ or lower (according to National Eye Institute criteria adapted by the Standardization of Uveitis Nomenclature Working Group; scores range from 0 to $4+$, with lower scores indicating greater severity of uveitis) [24]. Those intraocular inflammations were considered to be related to lenadogene nolparvovec in most instances. All occurrences of anterior chamber inflammation resolved without sequelae in response to standard topical antiinflammatory agents. Similarly, most incidences of vitritis responded to a short course of topical steroids, but two subjects required oral prednisone treatment (for a duration of approximately 2 months for the two subjects treated with $9 \times 10^{10} \mathrm{vg} /$ eye and $1.8 \times 10^{11} \mathrm{vg} /$ eye, respectively). One of those subjects who received the highest dose of lenadogene nolparvovec in cohort 4 experienced three severe episodes of uveitis flare (one episode of anterior chamber inflammation 
Fig. 1 Disposition of subjects. $D S M B$ data safety monitoring board, $v g$ viral genomes

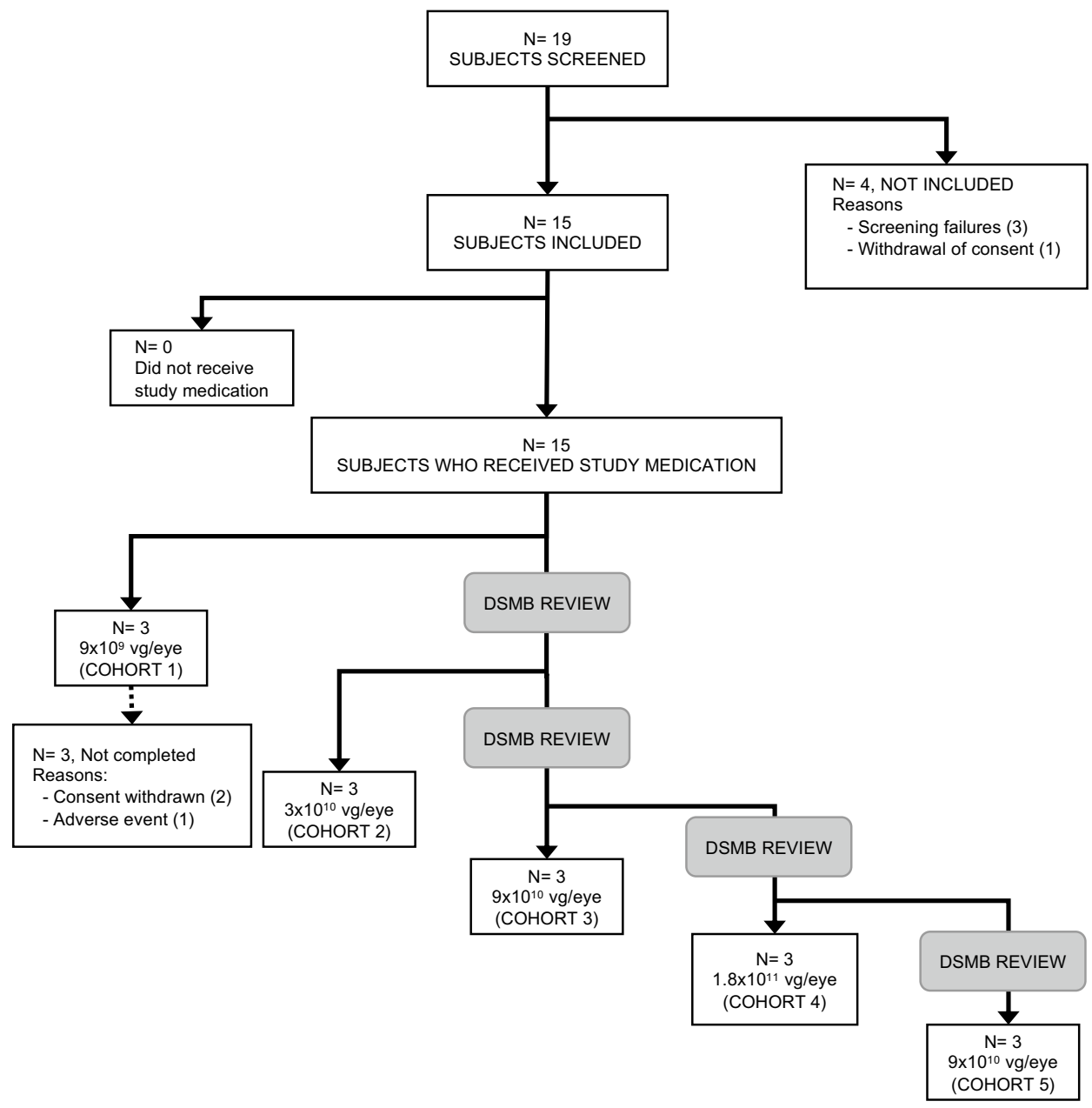

and two episodes of vitritis with vitreous haze). On this basis, the DSMB selected the $9 \times 10^{10} \mathrm{vg} /$ eye dose, which was one dose lower than highest dose of lenadogene nolparvovec, to take into the expansion cohort. None of these cases of intraocular inflammation involved any sequelae.

Elevation of IOP occurred in ten subjects (11 events). IOP elevations that were temporally related to the IVI injection (within $4 \mathrm{~h}$ post-dose) were considered by the investigator as due to the experimental procedure, whereas those recorded at later follow-up time points were considered as probably related to the study medication. Four events of ocular hypertension were related to study procedure and six were related to study medication. Most cases were mild, and all were transient and manageable without sequelae.

\subsubsection{Immune Response}

After unilateral IVI of lenadogene nolparvovec, a transient mild increase in serum AAV2 neutralizing antibodies (Nabs) titers was reported. This immune response was not dose dependent but was more related to the endogenous presence of anti-rAAV2 NAbs before drug administration. No association was observed between intraocular inflammation and humoral immune response.

Of the 15 subjects, 13 showed no cellular immune response during the time course of the study and two subjects did. However, for these two subjects, samples had been positive at baseline, suggesting that the rAVV2-specific cellular immune response measured could be due to a previous or concomitant AAV2 infection and not directly related to the treatment. No association was observed between intraocular inflammation and cellular immune response.

\subsubsection{Biodissemination}

In the REVEAL study, the quantified sequence was specific to the cytomegalovirus (CMV) promoter of the lenadogene nolparvovec vector. Viral shedding data showed that some tear samples were positive for the CMV promoter up to 1 week after lenadogene nolparvovec administration. No sample remained positive at week 2. The presence of lenadogene nolparvovec in blood was only detected in a few subjects and was mild, with levels close to the limit of 
quantification. Finally, all urine samples were negative for the presence of lenadogene nolparvovec.

\subsection{Efficacy}

\subsubsection{Best Corrected Visual Acuity—LogMAR}

Analyses of LogMAR BCVA in both treated and untreated eyes showed clinically meaningful improvements and were reflected in the mean change from baseline to year 5 for all subjects. For treated eyes, a mean improvement of LogMAR BCVA was noted for the entire study population and was - 0.44 LogMAR, equivalent to approximately 4 ETDRS lines (22 ETDRS letters). For the untreated eyes in the combined study population, a mean improvement in BCVA was - 0.49 LogMAR, equivalent to approximately 5 ETDRS lines (24.5 ETDRS letters). The improvements in the BCVA of treated and untreated eyes were maintained for the full 5-year follow-up period and are shown in Tables 1 and 2 . The mean \pm SD final BCVA was $\operatorname{LogMAR}+1.96 \pm 0.60$ and $+1.65 \pm 0.34$ in treated and untreated eyes, respectively. The best improvements in BCVA were seen for the $9 \times 10^{10}$ vg/eye dose previously selected by the DSMB as having the better benefit/risk, with a change of $-0.68 \operatorname{LogMAR}$, equivalent to approximately 7 ETDRS lines (34 ETDRS letters), and a change of $-0.64 \operatorname{LogMAR}$, equivalent to more than 6 ETDRS lines (32 ETDRS letters), in treated and untreated eyes, respectively. The improvements in the BCVA of treated and untreated eyes were maintained for the full 5-year follow-up period and are shown in Tables 1 and 2. The mean final BCVA was $\operatorname{LogMAR}+1.77 \pm 0.52$ and $+1.78 \pm 0.34$ in treated and untreated eyes, respectively.

Mean LogMAR BCVA for all subjects who completed the 5-year follow-up are shown graphically in Fig. 2, and the mean changes in these BCVAs from baseline for the treated eyes of subjects who completed the 5-year follow-up are summarized in Fig. 3. Mean values of LogMAR BCVA for both treated and untreated eyes in the combined cohorts that received the DSMB-selected dose of $9 \times 10^{10} \mathrm{vg} /$ eye are summarized in Fig. 4, and the mean changes in LogMAR from baseline in treated and untreated eyes in these same cohorts are illustrated in Fig. 5. There was no statistically significant difference between treated and untreated eyes at any visit during the 5 years of follow-up within any cohort or for all cohorts overall.

Table 1 Mean change of LogMAR from baseline to 5-year follow-up visit in treated eyes

\begin{tabular}{|c|c|c|c|c|c|}
\hline LogMAR & $\begin{array}{l}\text { Cohort } 1 \\
\left(9 \times 10^{9} \mathrm{vg} / \text { eye }\right) \\
(N=3)\end{array}$ & $\begin{array}{l}\text { Cohort } 2 \\
\left(3 \times 10^{10} \mathrm{vg} / \text { eye }\right) \\
(N=3)\end{array}$ & $\begin{array}{l}\text { Cohort } 4 \\
\left(1.8 \times 10^{11} \text { vg/eye }\right) \\
(N=3)\end{array}$ & $\begin{array}{l}\text { Cohorts } 3 \text { and } 5 \\
\left(9 \times 10^{10} \text { vg/eye }\right) \\
(N=6)\end{array}$ & $\begin{array}{l}\text { Total } \\
(N=15)\end{array}$ \\
\hline \multicolumn{6}{|l|}{ Baseline $^{\mathrm{a}}$} \\
\hline Number & 3 & 3 & 3 & 6 & 15 \\
\hline Mean \pm SD & $1.86 \pm 0.70$ & $2.47 \pm 0.76$ & $2.23 \pm 0.98$ & $2.45 \pm 0.70$ & $2.29 \pm 0.72$ \\
\hline $95 \%$ CI (mean) & $0.12-3.61$ & $0.58-4.35$ & $-0.20-4.65$ & $1.71-3.19$ & $1.89-2.69$ \\
\hline Median (Q1; Q3) & $2.01(1.10 ; 2.48)$ & $2.79(1.60 ; 3.01)$ & $2.79(1.10 ; 2.79)$ & $2.79(1.60 ; 3.01)$ & $2.79(1.60 ; 2.79)$ \\
\hline Minimum; maximum & $1.10 ; 2.48$ & $1.60 ; 3.01$ & $1.10 ; 2.79$ & $1.51 ; 3.01$ & $1.10 ; 3.01$ \\
\hline \multicolumn{6}{|l|}{ 5-year follow-up visit } \\
\hline Number & 0 & 3 & 3 & 6 & 12 \\
\hline Mean \pm SD & & $1.87 \pm 0.41$ & $2.44 \pm 0.81$ & $1.77 \pm 0.52$ & $1.96 \pm 0.60$ \\
\hline $95 \%$ CI (mean) & & $0.86-2.88$ & $0.42-4.45$ & $1.22-2.32$ & $1.58-2.34$ \\
\hline Median (Q1; Q3) & & $1.79(1.51 ; 2.31)$ & $2.79(1.51 ; 3.01)$ & $1.70(1.51 ; 1.79)$ & $1.79(1.51 ; 2.53)$ \\
\hline Minimum; maximum & & $1.51 ; 2.31$ & $1.51 ; 3.01$ & $1.20 ; 2.74$ & $1.20 ; 3.01$ \\
\hline \multicolumn{6}{|l|}{$\begin{array}{l}\text { Change from baseline to } \\
\text { 5-year follow-up visit }\end{array}$} \\
\hline Number & 0 & 3 & 3 & 6 & 12 \\
\hline Mean \pm SD & & $-0.60 \pm 0.57$ & $0.21 \pm 0.21$ & $-0.68 \pm 0.72$ & $-0.44 \pm 0.68$ \\
\hline $95 \%$ CI (mean) & & $-2.02-0.83$ & $-0.30-0.72$ & $-1.44-0.08$ & -0.87 to -0.01 \\
\hline Median (Q1; Q3) & & $-0.48(-1.22 ;-0.09)$ & $0.22(0.00 ; 0.41)$ & $-0.64(-1.00 ;-0.09)$ & $-0.18(-1.00 ; 0.05)$ \\
\hline Minimum; maximum & & $-1.22 ;-0.09$ & $0.00 ; 0.41$ & $-1.81 ; 0.09$ & $-1.81 ; 0.41$ \\
\hline
\end{tabular}

Improvement: $(5$ years - baseline $)<0$. Worsening: $(5$ years - baseline $)>0$

$C I$ confidence interval, LogMAR logarithm of the minimal angle of resolution, $Q$ quartile, $S D$ standard deviation, $v g$ viral genomes

${ }^{a}$ Baseline value is defined as the last available value measured prior to intravitreal injection (scheduled and unscheduled visits are considered) 
Table 2 Mean change of LogMAR from baseline to 5-year follow-up visit in untreated eyes

\begin{tabular}{|c|c|c|c|c|c|}
\hline LogMAR & $\begin{array}{l}\text { Cohort } 1 \\
\left(9 \times 10^{9} \text { vg/eye }\right) \\
(N=3)\end{array}$ & $\begin{array}{l}\text { Cohort } 2 \\
\left(3 \times 10^{10} \mathrm{vg} / \text { eye }\right) \\
(N=3)\end{array}$ & $\begin{array}{l}\text { Cohort } 4 \\
\left(1.8 \times 10^{11} \text { vg/eye }\right) \\
(N=3)\end{array}$ & $\begin{array}{l}\text { Cohorts } 3 \text { and } 5 \\
\left(9 \times 10^{10} \mathrm{vg} / \text { eye }\right) \\
(N=6)\end{array}$ & $\begin{array}{l}\text { Total } \\
(N=15)\end{array}$ \\
\hline \multicolumn{6}{|l|}{ Baseline $^{\mathrm{a}}$} \\
\hline Number & 3 & 3 & 3 & 6 & 15 \\
\hline Mean \pm SD & $1.77 \pm 0.49$ & $2.10 \pm 0.94$ & $1.70 \pm 0.61$ & $2.29 \pm 0.72$ & $2.03 \pm 0.68$ \\
\hline 95\% CI (mean) & $0.54 ; 2.99$ & $-0.24 ; 4.43$ & $0.19 ; 3.21$ & $1.53 ; 3.04$ & $1.65 ; 2.41$ \\
\hline Median (Q1; Q3) & $2.01(1.20 ; 2.09)$ & $1.60(1.51 ; 3.18)$ & $2.01(1.00 ; 2.09)$ & $2.29(1.60 ; 3.01)$ & $2.01(1.51 ; 2.79)$ \\
\hline Minimum; maximum & $1.20 ; 2.09$ & $1.51 ; 3.18$ & $1.00 ; 2.09$ & $1.51 ; 3.01$ & $1.00 ; 3.18$ \\
\hline \multicolumn{6}{|l|}{ 5-year follow-up visit } \\
\hline Number & 0 & 3 & 2 & 5 & 10 \\
\hline Mean \pm SD & & $1.60 \pm 0.20$ & $1.40 \pm 0.56$ & $1.78 \pm 0.34$ & $1.65 \pm 0.34$ \\
\hline 95\% CI (mean) & & $1.11-2.08$ & $-3.62-6.41$ & $1.36-2.20$ & $1.40-1.89$ \\
\hline Median (Q1; Q3) & & $1.60(1.40 ; 1.79)$ & $1.40(1.00 ; 1.79)$ & $1.79(1.60 ; 1.79)$ & $1.70(1.40 ; 1.79)$ \\
\hline Minimum; maximum & & $1.40 ; 1.79$ & $1.00 ; 1.79$ & $1.40 ; 2.31$ & $1.00 ; 2.31$ \\
\hline \multicolumn{6}{|l|}{$\begin{array}{l}\text { Change from baseline to } \\
\text { 5-year follow-up visit }\end{array}$} \\
\hline Number & 0 & 3 & 2 & 5 & 10 \\
\hline Mean \pm SD & & $-0.50 \pm 0.78$ & $-0.11 \pm 0.16$ & $-0.64 \pm 0.71$ & $-0.49 \pm 0.64$ \\
\hline $95 \%$ CI (mean) & & $-2.45-1.45$ & $-1.51-1.29$ & $-1.53-0.24$ & -0.95 to -0.04 \\
\hline Median (Q1; Q3) & & $-0.20(-1.39 ; 0.09)$ & $-0.11(-0.22 ; 0.00)$ & $-0.70(-1.00 ; 0.00)$ & $-0.21(-1.00 ; 0.00)$ \\
\hline Minimum; maximum & & $-1.39 ; 0.09$ & $-0.22 ; 0.00$ & $-1.61 ; 0.09$ & $-1.61 ; 0.09$ \\
\hline
\end{tabular}

Improvement: $(5$ years - baseline $)<0$. Worsening: $(5$ years - baseline $)>0$

$C I$ confidence interval, $\log M A R$ logarithm of the minimal angle of resolution, $Q$ quartile, $S D$ standard deviation, $v g$ viral genomes

${ }^{\text {a } B a s e l i n e ~ v a l u e ~ i s ~ d e f i n e d ~ a s ~ t h e ~ l a s t ~ a v a i l a b l e ~ v a l u e ~ m e a s u r e d ~ p r i o r ~ t o ~ i n t r a v i t r e a l ~ i n j e c t i o n ~(s c h e d u l e d ~ a n d ~ u n s c h e d u l e d ~ v i s i t s ~ a r e ~ c o n s i d e r e d) ~}$

Fig. 2 Mean LogMAR scores from baseline at each visit to year 5 for all treated eyes. Cohort 1: $9 \times 10^{9} \mathrm{vg} /$ eye; cohort 2: $3 \times 10^{10} \mathrm{vg} / \mathrm{eye}$; cohorts 3 and 5: $9 \times 10^{10} \mathrm{vg} /$ eye; cohort 4: $1.8 \times 10^{11}$ vg/eye. $\log M A R$ logarithm of the minimal angle of resolution, $v g$ viral genomes

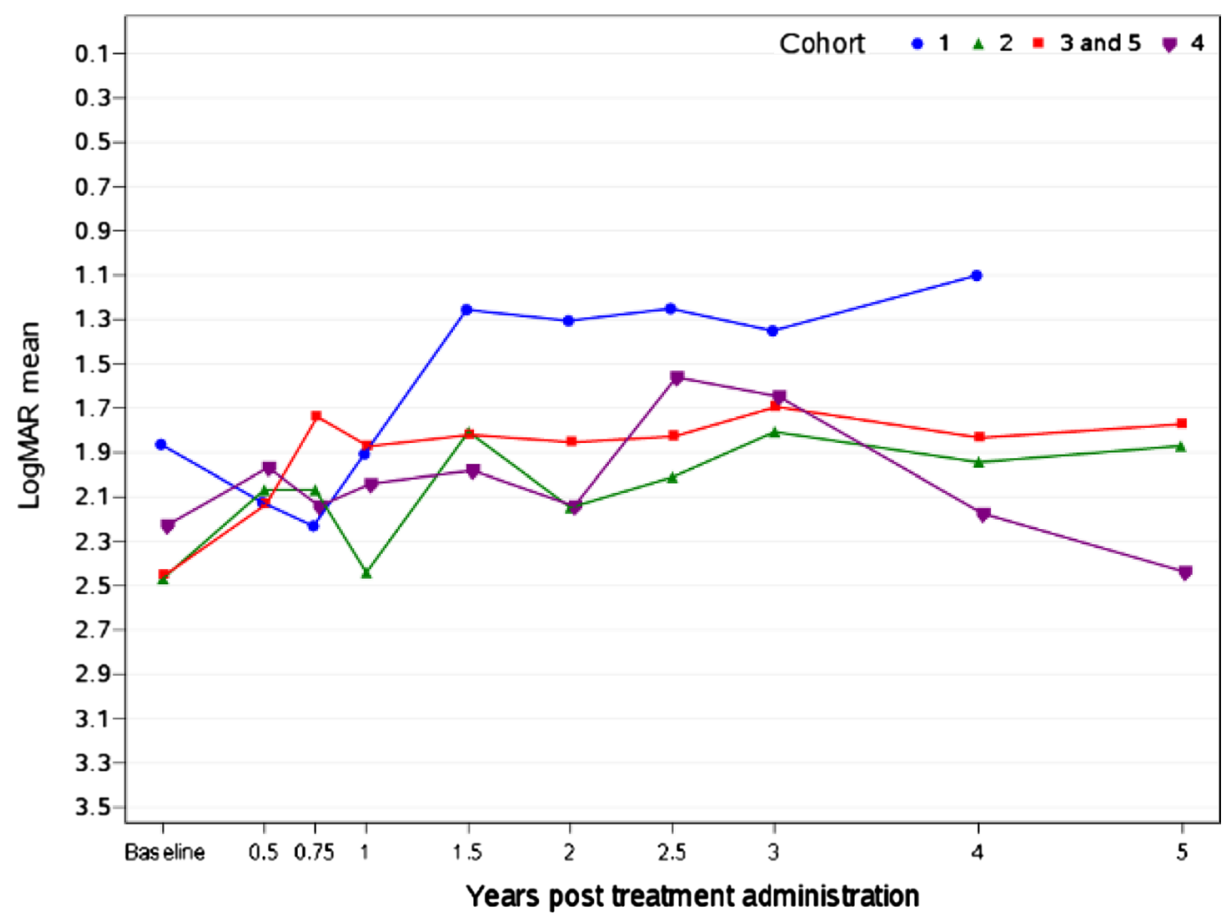


Fig. 3 Mean change in LogMAR from baseline at each visit to year 5 for all treated eyes. Cohort 1: $9 \times 10^{9} \mathrm{vg} /$ eye; cohort 2: $3 \times 10^{10} \mathrm{vg} / \mathrm{eye}$; cohorts 3 and 5: $9 \times 10^{10} \mathrm{vg} /$ eye; cohort 4: $1.8 \times 10^{11}$ vg/eye. LogMAR logarithm of the minimal angle of resolution, $v g$ viral genomes

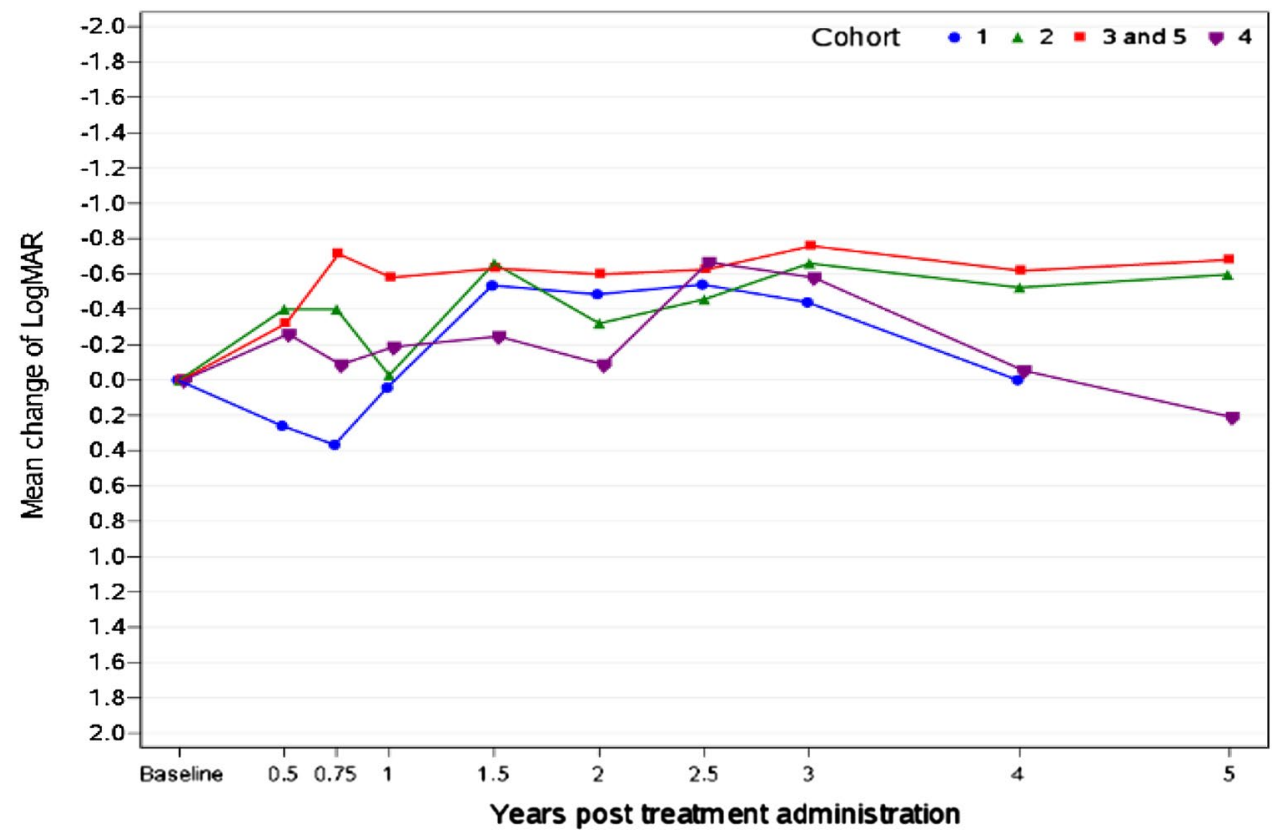

Fig. 4 Mean LogMAR scores at each visit and for both treated and untreated eyes at the $9 \times 10^{10} \mathrm{vg} /$ eye dose. LogMAR logarithm of the minimal angle of resolution, $v g$ viral genomes

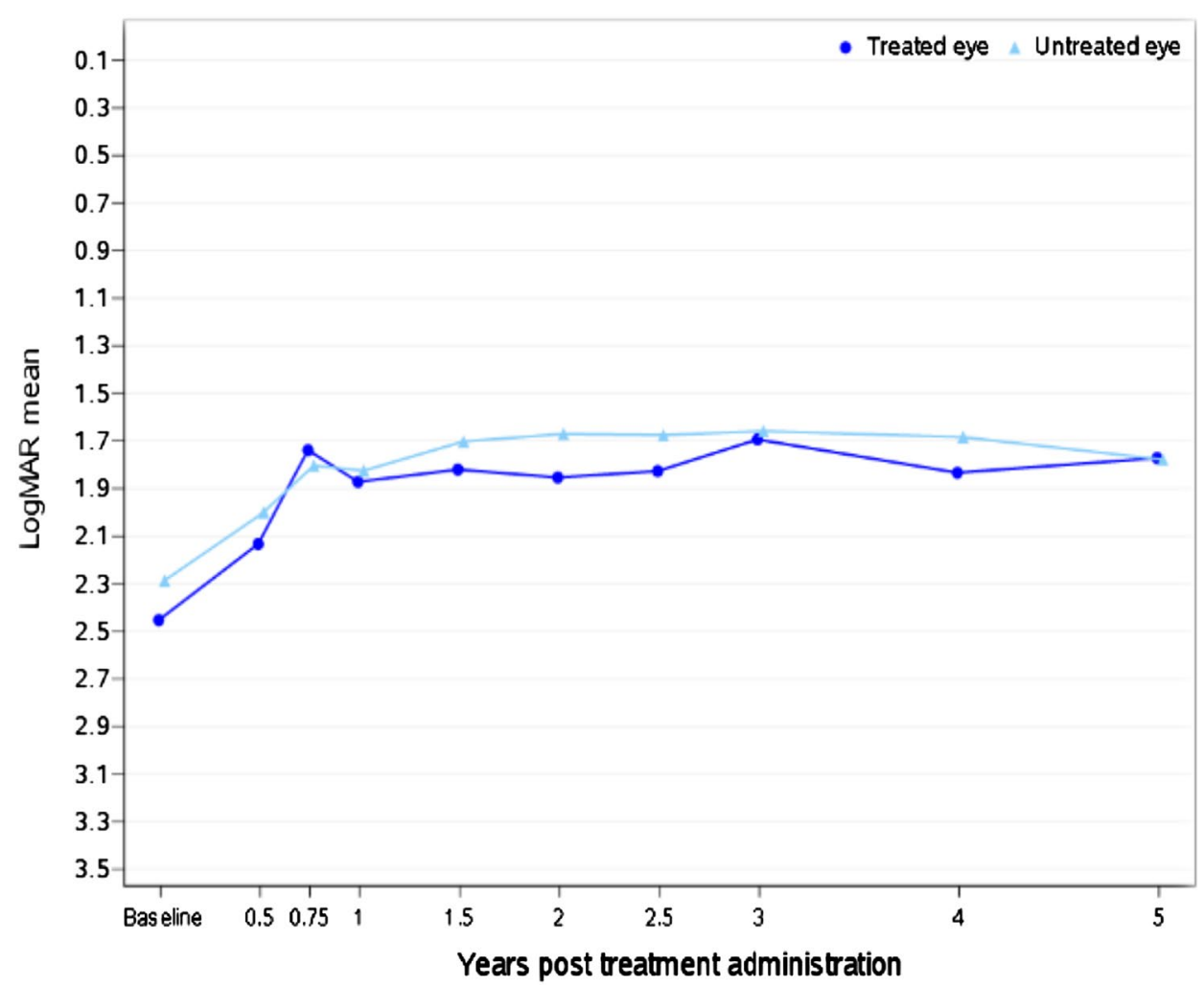

\subsubsection{Visual Field}

The measurement of visual field was performed with the Humphrey Visual Field (HVF) Analyzer using the SITA Standard 24-2 test (Stimulus white, size 3, fovea off). Mean deviation (MD) represents the average difference from normal values in the subjects' age group, and pattern SD (PSD) provides information about localized loss. Most HVFs were considered unreliable in relation to losses of fixation and false-positive or negative response rates. Eyes treated in cohorts 3 and 5, which were injected with the lenadogene nolparvovec dose of the pivotal phase III studies $\left(9 \times 10^{10} \mathrm{vg} /\right.$ eye $)$, showed a mean improvement of $2.66 \mathrm{~dB}$ in MD from baseline to the last follow-up. 
Fig. 5 Mean change of LogMAR at each visit for both treated and untreated eyes at the $9 \times 10^{10} \mathrm{vg}$ /eye dose. LogMAR logarithm of the minimal angle of resolution, $v g$ viral genomes

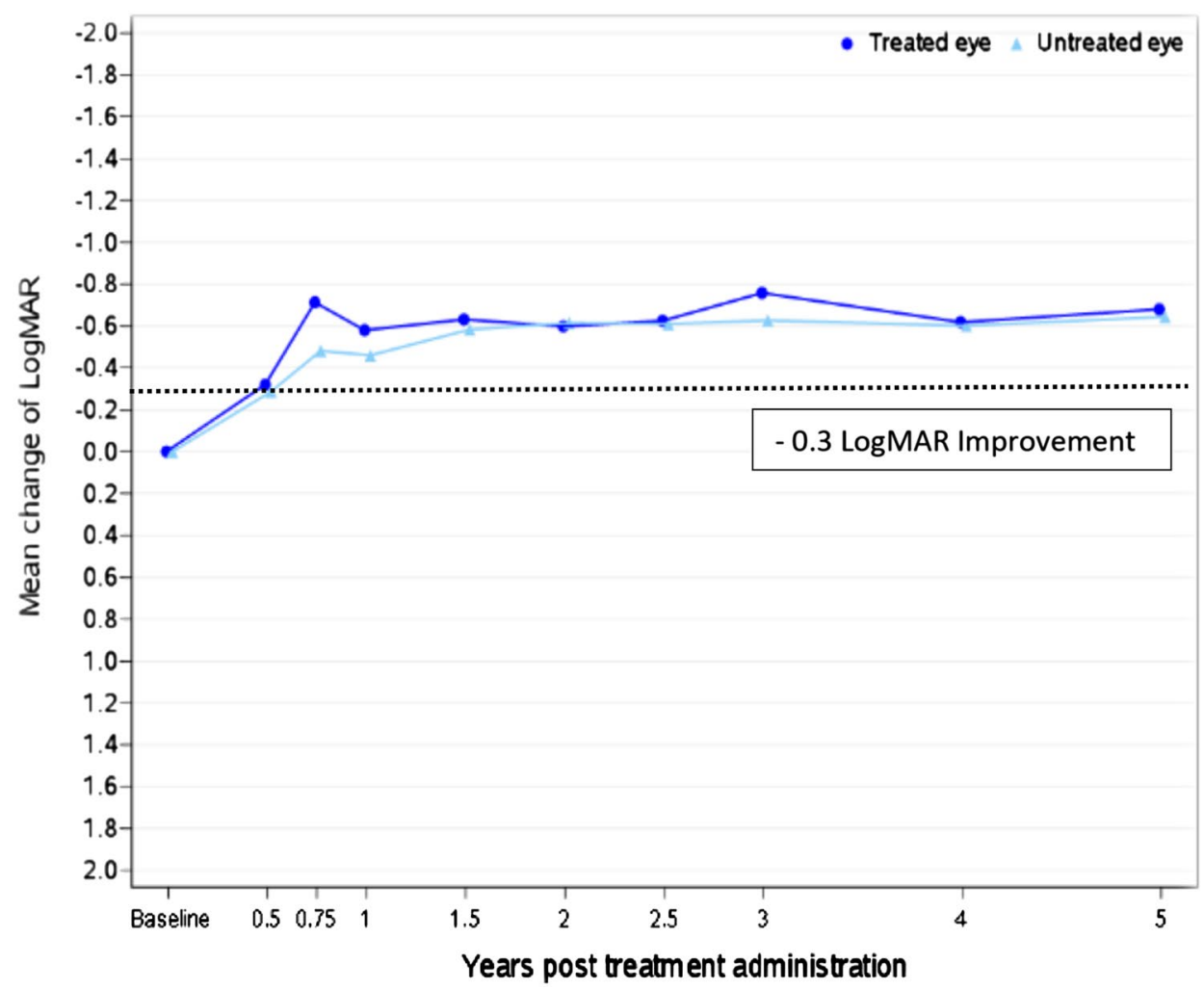

However, the mean PSD change from baseline was not clinically relevant in any of the cohorts, including cohorts 3 and 5. The treated eyes of subjects in cohorts 3 and 5 showed a mean improvement of $6.33 \mathrm{~dB}$ in foveal threshold values from baseline to the 5-year follow-up. Both these findings were in keeping with the improvements seen in visual acuity.

Octopus kinetic perimetry, which was used to measure central scotoma, and microperimetry were unreliable for most subjects and were only recorded for the first year of the study. Although Octopus kinetic perimetry showed a decrease in scotoma size for subjects who received $9 \times 10^{10}$ vg/eye during this initial study period, microperimetry showed no clinically relevant changes in these cohorts.

\subsubsection{Optical Coherence Tomography}

OCT was used to measure the thickness of the peripapillary RNFL, including the fibers of the papillomacular bundle, which is preferentially affected in LHON. When compared with healthy subjects of the same age range, baseline values for mean total RNFL thickness for treated eyes were low, with a mean value of $<50 \mu \mathrm{m}$ for all 15 subjects. For the entire study population, the treated eyes had a mean change from baseline to 5 years follow-up of $-7.92 \mu \mathrm{m}$.

\subsubsection{Other Secondary Endpoints}

Color vision (15 hue) and contrast sensitivity (Pelli Robson) were assessed during the initial 48-week study period and no improvements were recorded, although some patients volunteered improvement in perception of colors. Pattern VEPs were recorded during the same period and were found to be undetectable at all time points and dose levels. For most patients, flash VEPs were detectable at baseline and remained stable over 48 weeks. pERG was detected in three of the 15 subjects at baseline but undetectable in all subjects at week 48 .

\section{Discussion}

LHON classically manifests as acute to subacute bilateral painless central visual loss. The bilateral visual loss is typically sequential, and clinical manifestation in the first eye is essentially predictive of bilateral involvement [7, 9]. A recent prospective natural history study of subjects with the 11778 ND4 mutation reported that $53 \%$ of patients showed bilateral involvement within 2 months after onset in the first eye, and $80 \%$ reported bilateral involvement within 6 months [25]. Visual loss typically progresses to a nadir over a median of 6-8 weeks [9], and one study reported that $94 \%$ of subjects with available data reached nadir within 8 weeks of the onset of visual loss [10]. Mean time to visual 
stabilization was reported to be 3.7 months in a sample of 87 eyes [7].

Several retrospective studies have reported the final visual acuity outcomes in patients with LHON. At more than 24 months from onset of visual loss in subjects with the ND4 mutation, the median final acuity in the better-seeing eye was counting fingers (Snellen equivalent $20 / 1200$ ), with $73 \%$ of subjects qualifying for partial sightedness or legal blindness at outcome [9]. Nikoskelainen et al. [10] reported final visual acuities $<20 / 200$ in $73 \%$ of subjects with the 11778-ND4 mutation, and Oostra et al. [26] reported a mean final visual acuity of 20/182 in the better seeing eyes of patients with the 11778-ND4 mutation in another retrospective study, with only $19 \%$ of these eyes having a final visual acuity better than $20 / 200$. A prospective study of 44 subjects with ND4 LHON followed for up to 36 months reported a mean Snellen equivalent of $<20 / 320$ [25]. Furthermore, a meta-analysis of 204 subjects with $N D 4$ LHON, aged $\geq 15$ years at onset of the disease, revealed that spontaneous recovery was both rare and extremely poor when it did occur [18]. In addition, the prospective randomized placebo-controlled RHODOS study of idebenone showed no spontaneous recovery in visual acuity in any of the 22 control subjects during the 24 weeks of follow-up [20].

Subjects with ND4 LHON who are simply carriers of the 11778-ND4 mutation present a normal BCVA before the expression of the disease, and it has been shown that these non-affected patients with (asymptomatic) LHON (ND4 mutation carriers only) have a preserved BCVA (LogMAR 0 ; Snellen 20/20) [27, 28]. LHON is a debilitating condition with no symptomatic warnings of a rapid onset of visual loss. Most young people affected by ND4 LHON disease will become blind within 1 year. Irrespective of how the disease progresses, it inevitably results in bilateral visual loss with profound emotional and psychological impact on the affected individuals and those around them [29]. There is a clear unmet need in the medical management of patients with ND4 LHON. Therapeutic options for adolescent and adult patients with LHON are currently limited to idebenone, and its benefits in patients with $N D 4$ LHON are limited [19, 20].

As previously shown, spontaneous visual recovery in patients with LHON with the 11778-ND4 mutation is extremely rare and, when it does occur, the degree of improvement is minimal.

The efficacy signals seen in the REVEAL study are encouraging but have the following limitations: first, the REVEAL study was an open-label non-randomized uncontrolled study, where the eye with the worst vision was chosen for treatment. Neither the subjects nor the investigators and their study teams were masked to treatment, which inevitably resulted in bias with respect to BCVA measurement, which is a highly subjective assessment and dependent on how much effort a subject applies to the assessment. On the other hand, the fact that the study was conducted at a single center meant that the study assessments were homogeneous. It could also be argued that bias would have favored improvement in the treated eyes over the untreated eyes, but this was not the case.

Furthermore, the natural history of this disease would suggest that the improvements in BCVA observed in both the entire study population $(n=15)$ and in cohorts 3 and $5\left(9 \times 10^{10} \mathrm{vg} /\right.$ eye $)$ and maintained over a 5 -year period are unlikely to have occurred as the result of spontaneous recoveries. The best response to treatment was seen with the dose of $9 \times 10^{10} \mathrm{vg} /$ eye (cohorts 3 and 5), which was selected for the pivotal phase III studies and was clinically relevant (i.e., $\geq 0.3 \log M A R$ ) for both treated and untreated eyes starting 24 weeks after treatment administration and remaining stable up to the end of study (year 5) (Fig. 5). However, it should be highlighted that these 0.3 LogMAR improvements applied to "off-chart" assessments of visual acuity, with a mean \pm SD final $\operatorname{LogMAR}$ value of $+1.77 \pm 0.52$ and +1.78 \pm 0.34 for treated and untreated eyes, respectively. Therefore, despite meaningful improvements in LogMAR values, the final BCVA stayed off chart at 5 years post-treatment administration. These results from the REVEAL study are not as convincing as those seen in the two subsequent pivotal phase III studies (RESCUE and REVERSE; dose $9 \times 10^{10}$ vg/eye), when subjects were treated earlier during the course of their disease (all within 1 year of the onset of visual loss). In those two studies, the mean \pm SD final LogMAR value for all eyes (treated and sham eyes) was reported to be +1.36 \pm 0.60 using the last available observation (up to 4.3 years post-visual loss).

Binocular improvements in BCVA were seen in subjects following injection of a single eye with lenadogene nolparvovec. This contralateral therapeutic effect of lenadogene nolparvovec on untreated eyes was sustained, mirroring the improvement seen in the treated eyes, and this effect of lenadogene nolparvovec was further investigated in a nonclinical mechanistic study conducted in non-human primates (NHPs), demonstrating the transfer of viral vector DNA from the injected eyes to the uninjected contralateral eyes after unilateral lenadogene nolparvovec IVI [30]. Because viral vector DNA was detected and quantified in the optic chiasm in the monkey, it is suggested that the anatomic route taken by the viral vector DNA from the treated eye to the non-treated eye was via the optic nerve and chiasm, through anterograde and subsequent retrograde movement along the optic nerves. This pathway is supported by evidence of the transneuronal spread of adenovirus vector through synaptic transfer mechanisms [31]. A systemic transfer of lenadogene nolparvovec cannot be excluded, even if it is unlikely and less probable than interorbit transfer through 
the optic chiasm, as biodissemination studies have shown limited and transient presence of lenadogene nolparvovec in the blood. The expression in the non-treated eye of the ND4 protein, not assessed in our NHP study, would then explain the contralateral effect. Transfer of mitochondrial material (messenger RNA, proteins) cannot be excluded as a possible contributing mechanism of the visual improvement in the non-treated eye [32]. Finally, brain plasticity could account for an effect on visual function in the contralateral eye, as was shown for blind subjects implanted with retinal prosthesis [33].

Furthermore, the same contralateral effect of lenadogene nolparvovec was also observed in the two pivotal phase III studies (RESCUE and REVERSE) [30, 34]. A binocular improvement in BCVA has also been observed in other clinical development programs. Two gene therapies addressing subjects with ND4 LHON, using a viral vector, containing a complementary DNA coding for the human wild-type mitochondrial ND4 protein, have been studied in the USA [35, 36] and in China [37-40]. Both therapies administered the treatments intravitreally to a single eye, and both showed comparable bilateral improvements in vision [35-40].

The mean \pm SD disease duration was $4.6 \pm 8.7$ years, with a median of 1.1 years for the subjects who received the selected dose of $9 \times 10^{10} \mathrm{vg} / \mathrm{eye}$. Because of this long delay between the onset of visual loss and the time of lenadogene nolparvovec administration, the baseline BCVA should be considered as the final definitive BCVA value after nadir. Despite this long delay, BCVA did improve in four of the six subjects. Among these four subjects, two had experienced a visual loss within 1 year and two had experienced visual loss between 1 and 2 years prior to treatment (Table 3 ). Although the numbers of subjects studied are too small to allow us to draw definitive conclusions, these results suggest a potential benefit from gene therapy if subjects are treated within 2 years of visual loss.

None of the three visual field exams were found to be reliable in this study, which may be due to the dense central scotomas affecting the patients who were unable to complete the assessments. The same limitations also applied to the color vision, contrast sensitivity, pERG and VEP assessments. Likewise, OCT imaging did not prove to be a good marker for assessing the efficacy of gene therapy in this population because of the significant thinning of the RNFL already present at baseline, which showed little change over the 5-year follow-up period. This suggested a floor effect by the disease on the retinal nerve layers of interest.

These findings confirm that it was appropriate to select ETDRS BCVA as the primary endpoint for the subsequent pivotal phase III studies of lenadogene nolparvovec.

The REVEAL study was a dose-finding trial; the DSMB recommended that the highest dose of $1.8 \times 10^{11} \mathrm{vg} /$ eye given to the subjects was not sufficiently well tolerated. This recommendation was based on the observation that intraocular inflammation was more marked in the $1.8 \times 10^{11}$ $\mathrm{vg} /$ eye cohort than in the cohorts receiving lower doses. An extension cohort of three subjects was therefore treated with $9 \times 10^{10} \mathrm{vg}$ /eye, which was confirmed to be well tolerated and selected as the maximum tolerated dose to be taken into pivotal phase III studies.

In the REVEAL study, the immune response was limited and not dose dependent, indicating that lenadogene nolparvovec is not highly immunogenic when injected

Table 3 LogMAR best corrected visual acuity, changes from baseline to year 5 for treated and untreated eyes: safety population

\begin{tabular}{|c|c|c|c|c|c|c|c|}
\hline $\begin{array}{l}\text { Cohorts } 3 \text { and } 5 \\
\left(9 \times 10^{10} \mathrm{vg} / \text { eye }\right)\end{array}$ & Sex & Age, years & $\begin{array}{l}\text { Disease duration, } \\
\text { years }\end{array}$ & Eye & $\begin{array}{l}\text { Baseline } \\
\text { LogMAR }\end{array}$ & $\begin{array}{l}\text { Year } 5 \\
\text { LogMAR }\end{array}$ & $\begin{array}{l}\text { Change } \\
\text { from base- } \\
\text { line }\end{array}$ \\
\hline \multirow[t]{2}{*}{ Patient 01-009 } & Male & 71 & 22.21 & Left eye (U) & 1.51 & 1.6 & 0.09 \\
\hline & & & & Right eye (T) & 1.6 & 1.51 & -0.09 \\
\hline \multirow[t]{2}{*}{ Patient 01-011 } & Male & 29 & 1.36 & Left eye (U) & 2.79 & 1.79 & -1 \\
\hline & & & & Right eye (T) & 2.79 & 1.79 & -1 \\
\hline \multirow[t]{2}{*}{ Patient 01-012 } & Male & 59 & 1.58 & Left eye (T) & 2.79 & 1.79 & -1 \\
\hline & & & & Right eye (U) & 1.79 & 1.79 & 0 \\
\hline \multirow[t]{2}{*}{ Patient 01-017 } & Male & 44 & 0.88 & Left eye (T) & 3.01 & 2.74 & -0.27 \\
\hline & & & & Right eye (U) & 3.01 & 2.31 & -0.7 \\
\hline \multirow[t]{2}{*}{ Patient 01-018 } & Female & 51 & 0.79 & Left eye (T) & 1.51 & 1.6 & 0.09 \\
\hline & & & & Right eye (U) & 1.6 & & \\
\hline \multirow[t]{2}{*}{ Patient 01-019 } & Male & 38 & 0.58 & Left eye (T) & 3.01 & 1.2 & -1.81 \\
\hline & & & & Right eye (U) & 3.01 & 1.4 & -1.61 \\
\hline
\end{tabular}

Improvement: (visit value - baseline) $<0$. Worsening: (visit value - baseline) $>0$

$\log M A R$ logarithm of the minimal angle of resolution, $T$ treated eye, $U$ untreated eye, $v g$ viral genomes 
into the vitreous humor. Importantly, no association was observed between intraocular inflammation and humoral or cellular immune response [41]. In addition, the quantified sequence was specific to the CMV promoter of the lenadogene nolparvovec vector. Therefore, the few lowlevel positive results of lenadogene nolparvovec in the blood were potentially due to concomitant CMV infection and not necessarily to the presence of lenadogene nolparvovec in blood. Finally, biodissemination was limited in blood and tears and absent in urine.

The absence of any systemic issue related to lenadogene nolparvovec treatment is mainly supported by the limited biodissemination of the product, which was negligible in blood and tears and not detected in urine. Furthermore, the general humoral and cellular immunologic response was limited and not correlated with intraocular inflammation, confirming the local and ocular nature of the immune response. The most frequently reported ocular TEAEs consisted of intraocular inflammation, mostly mild in intensity, and localized to the anterior and intermediate segments. Intraocular inflammation was treated and controlled with local (topical) corticosteroids alone in most cases, with the exception of the highest dose, which was not pursued in subsequent phase III studies.

\section{Conclusion}

This study indicates that, after 5 years of follow-up, lenadogene nolparvovec was well tolerated, with a good overall safety profile. No unexpected AEs and no study drugrelated or study procedure-related SAEs occurred. No general safety issues were related to the study drug or the IVI procedure, and no TEAEs led to study discontinuation. The most frequently reported TEAEs were ocular, with intraocular inflammation and elevation of IOP being the most frequent. Increased IOP was mostly mild, well tolerated, and transient. Intraocular inflammation, localized in the anterior and intermediate segments, was mostly mild and responsive to standard topical anti-inflammatory treatment, with the exception of the highest dose, leading to the choice of a lower dose to be used in subsequent phase III studies. There were no sequelae from any of these events. Analysis of mean change of LogMAR in both treated and untreated eyes showed a clinically relevant and durable treatment effect for the 5 years post-treatment period, with the highest magnitude of effect for eyes injected with the pivotal phase III dose $\left(9 \times 10^{10} \mathrm{vg} /\right.$ eye $)$. However, the final mean BCVA remained "off chart" and was not at the level of improvement seen in subsequent pivotal phase III studies. The time between the onset of visual loss and treatment may play a role in the effect of gene therapy, and a therapeutic window of a maximum of 2 years between visual loss and treatment seems reasonable. There is a clear unmet medical need in the management of patients with ND4 LHON, and lenadogene nolparvovec offers this population the hope of a meaningful and sustainable improvement in vision following a simple one-time procedure.

Acknowledgements The authors are grateful to the study team that contributed to the conduct of REVEAL. We would also like to thank the patients who took part in this gene therapy study.

\section{Declaration}

Funding GenSight Biologics (Paris, France) fully funded and sponsored the study.

Author contributions All authors participated in the conceptualization, data collection, analysis, interpretation, writing, and critical review of the manuscript.

Conflicts of interest I Audo is supported by the Agence Nationale de la Recherche within the Programme Investissements d'Avenir, Institut Hospitalo Universitaire FOReSIGHT (ANR-18-IAHU-01), LabEx LIFESENSES (ANR-10-LABX-65), Retina France, UNADEV and Foundation Fighting Blindness. JA Sahel is supported by the Agence Nationale de la Recherche within the Programme Investissements d'Avenir, Institut Hospitalo Universitaire FOReSIGHT [ANR-18-IAHU-01] and LabEx LIFESENSES (ANR-10-LABX-65) and is a cofounder and shareholder of GenSight Biologics and a patent co-author on allotopic transport. C Vignal-Clermont is a consultant for Santhera Pharmaceuticals and GenSight Biologics. D O'Shaughnessy is a consultant for GenSight Biologics. M Taiel is employed by GenSight Biologics. JF Girmens, S Mohand Said, M-H Errera and L Plaine have no conflicts of interest that are directly relevant to the content of this article.

Ethics approval All procedures performed in the REVEAL clinical study involving human participants were in accordance with the ethical standards of the ethics committee and with the 1964 Helsinki Declaration and its later amendments or comparable ethical standards. The study was approved by the ethics committee "Comité de Protection des Personnes Ile de France V" (Hôpital Saint Antoine-184 rue du Faubourg Saint Antoine 75012 Paris) on 4 November 2013 and by the French Authorities (ANSM: Agence nationale de sécurité du médicament et des produits de santé) on 26 December 2013.

Consent to participate and publish Informed consent to participate in the study and for their data to be published was obtained from all participants.

Data and materials availability All data associated with this study are available in the main text.

Code availability Not applicable.

Open Access This article is licensed under a Creative Commons Attribution-NonCommercial 4.0 International License, which permits any non-commercial use, sharing, adaptation, distribution and reproduction in any medium or format, as long as you give appropriate credit to the original author(s) and the source, provide a link to the Creative Commons licence, and indicate if changes were made. The images or other third party material in this article are included in the article's Creative Commons licence, unless indicated otherwise in a credit line to the material. If material is not included in the article's Creative Commons 
licence and your intended use is not permitted by statutory regulation or exceeds the permitted use, you will need to obtain permission directly from the copyright holder. To view a copy of this licence, visit http://creativecommons.org/licenses/by-nc/4.0/.

\section{References}

1. Carelli V, Carbonelli M, de Coo IF, et al. International consensus statement on the clinical and therapeutic management of leber hereditary optic neuropathy. Neuro-Ophthalmol. 2017;37(4):371-81.

2. Wallace DC, Singh G, Lott MT, et al. Mitochondrial DNA mutation associated with Leber's hereditary optic neuropathy. Science. 1988;242(4884):1427-30.

3. Yu-Wai-Man P, Griffiths PG, Brown DT, et al. The epidemiology of leber hereditary optic neuropathy in the north east of England. Am J Hum Genet. 2003;72(2):333-9.

4. Yu-Wai-Man P, Votruba M, Burté F, et al. A neurodegenerative perspective on mitochondrial optic neuropathies. Acta Neuropathol. 2016;132(6):789-806.

5. Sadun A, La Morgia C, Carelli V. Leber's hereditary optic neuropathy. Curr Treat Opt Neurol. 2011;13(1):109-17.

6. Yu-Wai-Man P, Turnbull DM, Chinnery PF. Leber hereditary optic neuropathy. Am J Med Genet. 2002;39(3):162-9.

7. Newman NJ, Lott MT, Wallace DC. The clinical characteristics of pedigrees of Leber's hereditary optic neuropathy with the 11778 mutation. Am J Ophthalmol. 1991;111(6):750-62.

8. Koilkonda RD, Guy J. Leber's hereditary optic neuropathy-gene therapy. From benchtop to bedside. J Ophthalmol. 2011;2011:1-16.

9. Riordan-Eva P, Sanders MD, Govan GG, et al. The clinical features of Leber's hereditary optic neuropathy defined by the presence of a pathogenic mitochondrial DNA mutation. Brain. 1995;118(2):319-37.

10. Nikoskelainen EK, Huoponen K, Juvonen V, et al. Ophthalmologic findings in Leber hereditary optic neuropathy, with special reference to mtDNA mutations. Ophthalmology. 1996;103(3):504-14.

11. Majander A, Bowman R, Poulton J, et al. Childhoodonset Leber hereditary optic neuropathy. Br J Ophthalmol. 2017;101(11):1505-9.

12. Puomila A, Hämäläinen $P$, Kivioja $S$, et al. Epidemiology and penetrance of Leber hereditary optic neuropathy in Finland. Eur J Hum Genet. 2007;15(10):1079-89.

13. Spruijt L, Smeets HJ, Hendrickx A, et al. A MELAS-associated ND1 mutation causing Leber hereditary optic neuropathy and spastic dystonia. Arch Neurol. 2007;64(6):890-3.

14. Newman NJ. Hereditary optic neuropathies: from the mitochondria to the optic nerve. Am J Ophthalmol. 2005;140(3):517-23.

15. Newman NJ, Biousse V. Hereditary optic neuropathies. Eye. 2004;18(11):1144-60

16. Barboni P, Savini G, Valentino ML, et al. Leber's hereditary optic neuropathy with childhood onset. Investig Ophthalmol Vis Sci. 2006;47:5303-9.

17. Yu-Wai-Man P, Griffiths PG, Hudson G, et al. Inherited mitochondrial optic neuropathies. J Med Genet. 2009;46(3):145-58.

18. Newman NJ, Carelli V, Taiel M, et al. Visual outcomes in Leber hereditary optic neuropathy patients with the m.11778GA (MTDN4) mitochondrial DNA mutation. J Neuro-Ophthalmol. 2020. https://doi.org/10.1097/wno.0000000000001045.

19. EMA. European Public Assessment Report: Raxone. 2015 Jun 25. https://www.ema.europa.eu/en/documents/assessment-repor t/raxone-epar-public-assessment-report_en.pdf.
20. Klopstock T, Yu-Wai-Man P, Dimitriadis K, et al. A randomized placebo-controlled trial of idebenone in Leber's hereditary optic neuropathy. Brain. 2011;134(9):2677-86.

21. Marmor MF, Fulton AB, Holder GE, et al (for the International Society for Clinical Electrophysiology of Vision). ISCEV Standard for full-field clinical electroretinography (2008 update). Doc Ophthalmol. 2009;118:69-77.

22. McCulloch DL, Marmor MF. Brigell MG, et al. ISCEV Standard for full-field clinical electroretinography (2015 update). Doc Ophthalmol. 2015;130:1-12.

23. Jabs DA, Nussenblatt RB, Rosenbaum JT, et al. Standardization of Uveitis Nomenclature (SUN) Working Group. Standardization of uveitis nomenclature for reporting clinical data: results of the First International Workshop. Am J Ophthalmol 2005;140:509-516.

24. Nussenblatt RB, Palestine AG, Chan CC, et al. Standardization of vitreal inflammatory activity in intermediate and posterior uveitis. Ophthalmology. 1985;92:467-71.

25. Lam BL, Feuer WJ, Schiffman JC, et al. Trial End points and natural history in patients with G11778A Leber hereditary optic neuropathy: preparation for gene therapy clinical trial. JAMA Ophthalmol. 2014;132(4):428-36.

26. Oostra RJ, Bolhuis PA, Wijburg FA, et al. Leber's hereditary optic neuropathy: correlations between mitochondrial genotype and visual outcome. J Med Genet. 1994;31(4):280-6.

27. Guy J, Feuer WJ, Porciatti V, et al. Retinal ganglion cell dysfunction in asymptomatic G11778A: Leber hereditary optic neuropathy. Invest Ophthalmol Vis Sci. 2014;55(2):841.

28. Hwang 2017. Natural history of conversion of Leber's hereditary optic neuropathy ophthalmology Volume 124, Number 6, June 2017.

29. Kirkman MA, Korsten A, Leonhardt M, et al. Quality of life in patients with leber hereditary optic neuropathy. Invest Ophthalmol Vis Sci. 2009;50(7):3112-5.

30. Yu-Wai-Man P, Newman NJ, Carelli V, et al. Bilateral visual improvement with unilateral gene therapy injection for Leber hereditary optic neuropathy. Sci Transl Med. 2020;12:eaaz7423.

31. Zingg B, X.-L. Chou X.-L, Zhang Z.-G, L, et al. AAV-mediated anterograde transsynaptic tagging: Mapping corticocollicular input-defined neural pathways for defense behaviors. Neuron. 2017;93:33-47.

32. Cooper ML, Pasini S, Lambert WS, et al. Redistribution of metabolic resources through astrocyte networks mitigates neurodegenerative stress. Proc Natl Acad Sci USA. 2020;117(31):18810-21.

33. Castaldi E, Cicchini GM, Cinelli L, Biagi L, Rizzo S, Morrone MC. Visual BOLD response in late blind subjects with argus II retinal prosthesis. PLoS Biol. 2016;14(10):e1002569.

34. Newman NJ, Yu-Wai-Man P, Carelli V, et al. Efficacy and safety of intravitreal gene therapy for Leber hereditary optic neuropathy treated within 6 months of disease onset. Ophthalmology. 2020;. https://doi.org/10.1016/j.ophtha.2020.12.012.

35. Feuer WJ, Schiffman JC, Davis JL, et al. Gene therapy for leber hereditary optic neuropathy: initial results. Ophthalmology. 2016;123(3):558-70.

36. Guy J, Feuer WJ, Davis JL, et al. Gene therapy for Leber hereditary optic neuropathy: low- and medium-dose visual results. Ophthalmology. 2017;124(11):1621-34.

37. Yuan J, Zhang Y, Liu H, et al. Seven-year follow-up of gene therapy for Leber's hereditary optic neuropathy. Ophthalmology. 2020;127(8):1125-7.

38. Liu HL, Yuan JJ, Zhang Y, et al. Factors associated with rapid improvement in visual acuity in patients with Leber's hereditary optic neuropathy after gene therapy. Acta Ophthalmol. $2020 \mathrm{Feb}$ 24. https://doi.org/10.1111/aos.14379. [Epub ahead of print].

39. Yuan J, Zhang Y, Wang L, et al. Visual field variability after gene therapy for Leber's hereditary optic neuropathy. Ophthalm Res. 2018;60(3):176-84. 
40. Yang S, Ma S, Wan X, et al. Long-term outcomes of gene therapy for the treatment of Leber's hereditary optic neuropathy. EbioMedicine. 2016;10:258-68.

41. Bouquet $\mathrm{C}$, Vignal Clermont $\mathrm{C}$, Galy A, et al. Immune response and intraocular inflammation in patients with Leber hereditary optic neuropathy treated with intravitreal injection of recombinant adeno-associated virus 2 carrying the ND4 gene: a secondary analysis of a phase 1/2 clinical trial. JAMA Ophthalmol. 2019;137(4):399. 